\title{
CAUSATION AND CAUSAL SELECTION IN THE BIOPSYCHOSOCIAL MODEL OF HEALTH AND DISEASE
}

\author{
Hane Htut Maung ${ }^{1}$ \\ ${ }^{1}$ University of Manchester
}

Original scientific article - Received: 27/01/2021 Accepted: 05/05/2021

\section{ABSTRACT}

In The Biopsychosocial Model of Health and Disease, Derek Bolton and Grant Gillett argue that a defensible updated version of the biopsychosocial model requires a metaphysically adequate account of disease causation that can accommodate biological, psychological, and social factors. This present paper offers a philosophical critique of their account of biopsychosocial causation. I argue that their account relies on claims about the normativity and the semantic content of biological information that are metaphysically contentious. Moreover, I suggest that these claims are unnecessary for a defence of biopsychosocial causation, as the roles of multiple and diverse factors in disease causation can be readily accommodated by a more widely accepted and less metaphysically contentious account of causation. I then raise the more general concern that they are misdiagnosing the problem with the traditional version of the biopsychosocial model. The challenge when developing an explanatorily valuable version of the biopsychosocial model, I argue, is not so much providing an adequate account of biopsychosocial causation, but providing an adequate account of causal selection. Finally, I consider how this problem may be solved to arrive at a more explanatorily valuable and clinically useful version of the biopsychosocial model.

Keywords: Derek Bolton; Grant Gillett; biopsychosocial model; causation; causal selection 


\section{Introduction}

The biopsychosocial model, initially developed by George Engel (1977), is perhaps the most widely accepted model of health and disease in contemporary medicine. As the name suggests, the model emphasises the importance of considering biological, psychological, and social dimensions of health and disease in clinical practice. In recent years, however, the model has recently been criticised for being too vague to have any explanatory value or predictive power. The psychiatrist Nassir Ghaemi, for example, has suggested that the biopsychosocial model is not a scientific model, but is little more than "a slogan whose ultimate basis was eclecticism (...) meant to free practitioners to do what they pleased" (Ghaemi, 2010, p. 213).

Responding to this criticism, Derek Bolton and Grant Gillett aim to develop a defensible version of the biopsychosocial model that can support the theory and practice of contemporary medicine. In The Biopsychosocial Model of Health and Disease (2019), they propose that an appropriately updated version of the model can provide a philosophical framework which facilitates the understanding of disease causation. Given the increasing evidence that psychological and social factors have important roles in disease causation, they argue that physicalistic reductionism is false and that some version of the biopsychosocial model is required in medicine. However, a problem with the traditional version of the biopsychosocial model is that it does not tell us how these biological, psychological, and social factors interact causally. Accordingly, they suggest that a suitably updated version of the model must include a metaphysically adequate account of biopsychosocial causation that can accommodate the roles of these multiple and diverse factors.

In this paper, I offer a philosophical critique of the analysis of biopsychosocial causation provided by Bolton and Gillett. While I agree with them that physicalistic reductionism is untenable and that some version of the biopsychosocial model is warranted, I argue that their causal approach to defending the model is problematic. In $\$ 2$, I briefly lay out the account of biopsychosocial causation provided by Bolton and Gillett. In $\S 3$, I show that their account relies on claims about the normativity and the semantic content of biological information that are metaphysically contentious. Moreover, I suggest that these claims are unnecessary for a defence of biopsychosocial causation, as the roles of multiple and diverse factors in disease causation can be readily accommodated by a more widely accepted and less metaphysically contentious account, namely James Woodward's (2004) interventionist theory of causation. In $\S 4$, I raise a more general worry, which is that Bolton and Gillett are misdiagnosing the 
problem with the traditional version of the biopsychosocial model. The key challenge when developing an explanatorily valuable version of the biopsychosocial model, I suggest, is not so much providing a metaphysically adequate account of causation, but providing an epistemically useful account of causal selection. That is to say, the vagueness of the biopsychosocial model is related to its inability to tell us which causal factors, out of the vast network of biological, psychological, and social factors, are explanatorily significant. Finally, I consider how this problem may be solved to arrive at a more explanatorily valuable and clinically useful version of the biopsychosocial model.

\section{An Account of Biopsychosocial Causation}

The traditional version of the biopsychosocial model presented by Engel (1977) arose in response to the prevailing model in medicine at the time, which was the biomedical model of health and disease. This is characterised as follows:

It assumes disease to be fully accounted for by deviations from the norm of measurable biological (somatic) variables. It leaves no room within its framework for the social, psychological, and behavioral dimensions of illness. The biomedical model not only requires that disease be dealt with as an entity independent of social behavior, it also demands that behavioral aberrations be explained on the basis of disordered somatic (biochemical or neurophysiological) processes. (Engel 1977, 130)

A key feature of the biomedical model, then, is physicalistic reductionism, or the assumption that disease can be reductively explained at the lowest biological level, which may be biochemical or neurophysiological. Psychological and social factors are either excluded from the explanation or assumed to be reducible to processes at the biological level.

While the biomedical model is supported by advances in biomedical science, Engel argues that it has serious limitations that make it insufficient as a general model for medicine. These include its neglect of the patient's account of the illness, its inability to consider how social circumstances influence the presentations and meanings of health and disease, and its failure to acknowledge the roles of psychological and social factors in disease causation. In their book, Bolton and Gillett spend considerable time on the last of these, citing the accumulating evidence that psychological and social factors have causal roles in health and disease. They list a wide range of conditions that are influenced by psychological and social factors: 
For example: breast cancer (...) atopic disease, generally, including for asthma; HIV and musculoskeletal disorders. In addition, psychosocial factors have been implicated in outcomes of surgical procedures, for example, chronic pain; lumbar and spinal surgery; liver transplant (...) and coronary artery bypass (...) In addition, there is evidence for psychosocial factors in wound healing, and extent of fatigue after traumatic brain injury. Psychosocial factors have also been implicated in responses to other interventions for medical conditions, such as inpatient rehabilitation for stroke patients (...) and effects of hospitalisation on older patients. (Bolton and Gillett 2019, 11-12)

The above is supported by the extensive epidemiological research of Michael Marmot (2005), who demonstrated robust correlations between social statuses and the incidences of a wide range of medical conditions. Hence, just as the biomedical model is of interest because of the advances in biomedical science, the biopsychosocial model is supported by advances in psychology, epidemiology, and social science.

In the present day, the contributions of psychological and social factors are especially apparent in the increasing rates of mental health problems in young people. Bolton explores some of these factors in a recent paper coauthored with the psychiatrist Dinesh Bhugra (Bolton and Bhugra, 2020). They argue that changes in society over the past few decades have contributed to worsening mental health problems among children, adolescents, and young adults. For example, due to the development of social media and the public profile of populism, political conflicts between conservatives and liberals have become more visible and pervasive in ways that have eroded the shared norms of rationality in political discourse and have resulted in the loss of social cohesion. Moreover, due to government austerity, neoliberal financialisation, and economic downturn, intergenerational wealth inequalities have increased, with young adults from the millennial generation having less stable accommodation, less career certainty, and less financial security than older adults from the baby boomer generation. The negative mental health effects of these economic and political factors are corroborated by epidemiological data showing that invoking government austerity during an economic recession increases the population suicide rate, while investing in social welfare during an economic recession does not have this outcome (Stuckler and Basu, 2013). Finally, younger generations are also affected by serious concerns regarding anthropogenic climate change and the inadequate geopolitical response to the environmental crisis. 
Given that neither the genetic nor the neurobiological characteristics of people have changed significantly over the past few decades, the biomedical model appears inadequate to account for these increasing rates of mental health problems in young people. Rather, Bolton and Bhugra (2020) argue that a broad biopsychosocial approach is required to account for the contributions of the aforementioned changes in society to these worsening mental health problems. Accordingly, in their book, Bolton and Gillett (2019) develop a metaphysical account of causation that avoids the physicalistic reductionism of the biomedical model and accommodates the roles of biological, psychological, and social factors in disease causation.

Against physicalistic reduction, Bolton and Gillett argue that explanations in biology are irreducible to explanations in chemistry and physics. Following the work of Erwin Schrödinger (1944), they suggest that biological systems are characterised by their abilities to extract energy from the environment and resist local increases in entropy, thus allowing them to maintain stable forms, develop in ordered ways, and reproduce. According to Bolton and Gillett, biological systems can do this because they use information transfer to control energy transfer. They write:

Physical and chemical processes involve energy transfers covered by mathematical energy equations, but in biological organisms the physical and chemical processes not only happen, but can only happen in the right place at the right time in the right degree, if there are mechanisms that control and regulate them in a way appropriate to bringing about a particular function. (Bolton and Gillett 2019, 48)

The informational nature of biological causation, Bolton and Gillett argue, is irreducible to physical explanation, because it involves semantic content. The dynamics of this semantic content follow regularities that are not captured by the lawlike regularities of physics and chemistry. Bolton and Gillett continue:

Another way of making this point is that the energy transfer involved in information transfer is irrelevant to the information transfer. The flow of information depends on regularities, but these regularities are not determined by the energy equations of physics and chemistry, rather they must rely on other properties of materiality. The concept required at this point is expressed by such terms as structure, form, shape or syntax (to borrow from logic) — that codes information. (Bolton and Gillett 2019, 49) 
For example, sequences of nucleotides on genes encode information that is used by intracellular components to construct proteins, patterns of action potentials in neurons encode information that influence how neurotransmitters are secreted, and ligands encode information in virtue of their selective interactions with receptors.

Bolton and Gillett go on to argue that the semantic content of biological information makes biological causation normative and teleological. That is to say, there are "right" and "wrong" ways for the semantic content to be decoded, which pertain respectively to whether or not they are conducive to the biological systems fulfilling their goals or functions. Such normativity, Bolton and Gillett suggest, makes causation in biology different from causation in physics. While causation in biology is characterised by the capacity for error, causation in physics is purported to follow laws and equations that cannot be violated. They write:

The general conceptual point at issue here is that regulation and control mechanisms keep things going right rather than wrong. Such normativity is not present in the energy equations of physics and chemistry, which always apply and never fail. It arises in biology for the first time, marking a fundamental departure of biology from physical and chemical processes alone. The normativity is implied in all of the key systems theoretic concepts such as regulation, control and information. It derives from the point that biological systems function towards ends, and function well and badly accordingly as they do or do not attain them. (Bolton and Gillett 2019, 51)

For example, at the genetic level, the sequences of nucleotides are usually conserved during genetic replication, but mutations occasionally occur due to "replication errors", some of which can have harmful effects for the organisms. At the molecular level, immunoreceptors usually bind selectively with particular foreign ligands, but occasionally they react with antigens from hosts due to "molecular mimicry", which can be associated with autoimmune reactions. At the organismal level, a behaviour, such as feeding, is usually adaptive insofar as it contributes to the survival and reproduction of the organism, but occasionally may be maladaptive, such as when it leads to the ingestion of a toxin.

Informational content and normativity are also characteristics of psychological and social processes. For example, perception can be deemed accurate or inaccurate according to perceptual norms, belief can be deemed rational or irrational according to epistemic norms, speech may be deemed correct or incorrect according to linguistic norms, and 
behaviour can be deemed permissible or impermissible according to moral, legal, and social norms. Bolton and Gillett suggest that these interact with the informational content and normativity of biological processes through embodied agency. They draw on a recent development in the philosophy of mind, which Albert Newen, Leon De Bruin, and Shaun Gallagher call 4E cognition (Newen et al. 2018). This proposes that cognition has the four following features:

1. 'Embodied' (in the body)

2. 'Embedded' (in the environment; in causal loops with it)

3. 'Enactive' (Acting in and manipulating the environment, directly, not via a representation or model; the environment offers affordances, or opportunities, for action and manipulation)

4. 'Extended' (Extended to the body and environment, including devices used for cognitive functioning). (Bolton and Gillett 2019, 78)

Psychological agency, according to Bolton and Gillett, is embodied in the biological body and, in virtue of the informational transfer that occurs in the biological body, is an active causal power whose influence extends into the social environment. Accordingly, normative processes at biological, psychological, and social levels can interact with one another causally via the regulatory flow of information.

To bring this all together, let us see how it might apply to the aforementioned increasing rates of mental health problems among young people (Bolton and Bhugra, 2020). Recent social and political changes, including the shared norms of rationality in political discourse being undermined, increasing intergenerational wealth inequalities, and escalating concerns about anthropogenic climate change, lead to adverse social conditions. These have downward regulatory effects that restrict psychological agency, constrain how biological resources are distributed, and disrupt the usual flow of information in the biological system. In turn, the alteration in the informational transfer in the biological system further affects psychological agency and disrupts how the person interacts with the social environment, manifesting in mental ill health.

Here, the biological, psychological, and social processes are integrated, with information transfer being the common currency in the causal interactions across these three domains. This information transfer has a normative dimension that is irreducible to the sort of causal explanation that features in physics. And so, the account of biopsychosocial causation developed by Bolton and Gillett (2019) accommodates the roles of 
multiple and diverse factors in disease causation while avoiding the physicalistic reductionism of the biomedical model. However, their account relies on claims about the normativity and semantic content of biological information that are metaphysically contentious. In the following section, I examine some of the problems with these claims and show that they are unnecessary for an adequate account of biopsychosocial causation.

\section{Critical Discussion}

Bolton and Gillett are indeed correct that informational content and normativity are properties of the psychological and social domains respectively. Psychological agency is marked by intentionality and meaning, which are embedded in the wider social context and appear to be irreducible to the regularities studied in physics. The social environment is marked by our values, norms, and conventions, which regulate our behavioural affordances, interpersonal interactions, and communicative practices. Hence, informational content and normativity in the psychological and social domains have their sources in our intentions, values, interests, and judgements at the interpersonal level. However, claiming that normativity and informational content are properties of the biological domain at the subpersonal level is more problematic. Of course, Bolton and Gillett are correct that we often use normative and informational notions, such as function, dysfunction, sense, and error, in biological theorising. The problem, though, is that these normative and informational notions may be features that we project onto biological processes, rather than intrinsic properties of the biological processes themselves. That is to say, we derive notions from our understandings of the genuine normativity and informational content of the social and psychological domains, and then we use these notions as instrumental metaphors to organise our theoretical thinking about biological processes.

The above presents challenge to the account of biopsychosocial causation presented by Bolton and Gillett for the following reason. As noted above, information transfer is supposed to be the common currency in the causal interactions across biological, psychological, and social domains. However, if normativity and informational content are not genuine properties of biological causation but are merely instrumental metaphors that we use to organise our theoretical thinking about biological processes, then such information transfer cannot comprise the common currency that is conserved across the three domains in biopsychosocial causation. Causation in the psychological and social domains may involve genuine 
normative and informational properties, but it is doubtful whether these properties can actually be said to be conserved at the biological level.

My contention that normative and informational notions in biology are instrumental metaphors can be illustrated in two ways. First, I consider how mechanical laws and explanations in physics might be rephrased in teleological and normative terms. This challenges the claim by Bolton and Gillett that normativity is what makes causation in biology different from causation in physics. Second, I consider how explanations in biology that invoke normative and informational notions might be rephrased in terms that are more descriptive. This challenges the claim that normativity and informational content are intrinsic properties of the biological processes themselves.

With respect to causation in physics, recall that Bolton and Gillett claim that this follows laws and equations that cannot be violated, in contrast with causation in biology which they claim is capable of error. However, the regularities in physics may not be as faultless as Bolton and Gillett suggest. Suppose, for example, that a trolley with a known mass is attached to a hanging stone of a known weight via a pulley and the acceleration of the trolley is measured. The theoretical law in this case is $F=m \times a$, where $F$ is the total pulling force of the hanging weight, $m$ is the mass of the trolley, and $a$ is the acceleration of the trolley. Now, if the experiment is repeated under a variety of background conditions, $a$ may turn out not to be the same in each instance despite $F$ and $m$ being kept constant. That is to say, the observations may deviate from what is predicted by $F=m \times a$ in different ways.

As noted by Imre Lakatos (1974), when this happens, we tend to invoke auxiliary hypotheses which introduce other variables, in order to conserve $F=m \times a$. For example, we may try to explain the variability in $a$ across the different experimental conditions by considering possible confounding factors, including variations in the energy lost through friction, air resistance, and elasticity of the cord attaching the trolley to the weight. However, our hypotheses based on these confounding factors may not be able to yield quantities that are sufficiently exact to conserve $F=m \times a$. Indeed, as Nancy Cartwright (1983) points out, solving the derived equations to see whether or not they fit with our observations may be mathematically intractable. For example, if we try to derive the energy lost through friction from the mechanical and thermodynamic properties of the trolley and the surface, and then try to predict how this would affect the movement of the trolley at different moments in its trajectory, we may only yield rough approximations. Hence, far from being faultless, the 
regularities in physics are associated with various deviations for which we may not be able to account mathematically.

This capacity for error in physics raises the possibility of rephrasing mechanical laws and explanations in teleological and normative terms, akin to explanations in biology. To take another example, consider the law that a system comprising two objects in contact with each other will proceed toward thermal equilibrium. This can be rephrased as a teleological and normative claim, whereby proceeding towards the "goal" of thermal equilibrium is what the system "should" do. However, in actuality, systems tend not to be closed, and so may involve thermal disequilibria that deviate from this law. These could be interpreted as cases where contingent circumstances result in the systems "failing" to proceed as they "should", analogous to dysfunctions in biological systems. An objection might be to say that while there can be localised thermal disequilibria, the universe as a whole is proceeding toward thermal equilibrium, which will eventually result in these localised thermal disequilibria being dissipated. In response, though, an analogous claim could be made regarding dysfunctions in biological systems. That is to say, while there can be localised dysfunctions that compromise the survival and reproductive prospects of organisms, but it could be claimed that the frequencies of these dysfunctions will eventually diminish through the process of natural selection.

Of course, these teleological and normative notions are not intended to be literal. That is to say, they involve no ontological commitment to the claim that systems in physics actually have "goals". Rather, they are instrumental metaphors that are derived from the teleological and normative notions we use in the psychological and social domains, which concern our intentions, values, interests, and judgements. Nonetheless, the possibility of rephrasing regularities in physics in teleological and normative terms suggests that they may not necessarily be so different from regularities in biology. It gives us grounds to consider whether the teleological and normative notions in biological explanations are also instrumental metaphors, rather than being representations of actual properties of biological processes. To be clear, this is not to say that biological explanation can be reduced to physical explanation. I agree with Bolton and Gillett that the complex causal processes in biology are not straightforwardly reducible to the mechanical laws and explanations in physics. Rather, it is to say that the difference between the domains of biology and physics cannot be captured by the presence or absence of normativity. This can be further demonstrated by examining how teleological and normative explanations in biology can be rephrased in terms that are more descriptive. 
With respect to causation in biology, recall that Bolton and Gillett claim that this is characterised by informational content that can be decoded in "right" or "wrong" ways, which pertain respectively to whether or not they are conducive to the biological systems fulfilling their goals or functions. At the genetic level, they suggest that information is encoded in the sequences of nucleotides on chromosomes and, if decoded properly, contributes to the proper forms of the biological systems being maintained. Here, Bolton and Gillett seem to adhere to the modern evolutionary synthesis, which considers the genome to be a "blueprint" for the realisation of the phenotype (Plomin, 2018). A notable proponent of this view is Richard Dawkins, who suggests that the "information passes through bodies and affects them, but it is not affected by them on its way through" (Dawkins 1995, 4).

However, recent developments in the philosophy of biology have undermined the modern evolutionary synthesis. An important contribution is a theoretical framework, put forward by Susan Oyama, Paul Griffiths, and Russell Gray, called developmental systems theory (Griffiths and Gray, 1994; Oyama, 2000). Developmental systems theory emphasises that the genome is just one among many dynamic resources that interact to produce a phenotypic outcome, including epigenetic modifications, transcription factors, intracellular reactions, physiological processes, nutritional resources, environmental conditions, social interactions, and cultural contexts. That is to say, the phenotype is not the inevitable realisation of a genetic "blueprint", but is the contingent outcome of complex and dynamic interactions between multiple resources, some of which may also be inherited across generations. Variations in these resources can result in variations in the phenotypic outcomes. Accordingly, Griffiths and Gray (1994) argue that the genome cannot be considered to be a unique bearer of developmental information. Given that the particular causal role of the genome is contingent on the state of the rest of the developmental system, it makes just as much sense to say that the rest of the developmental system encodes information that is "read" by the genome as it does to say that the genome encodes information that is "read" by the rest of the developmental system. Informational content, then, is not an intrinsic property of biological causation, but is an instrumental metaphor whose application depends on what part of the developmental system we decide to hold fixed. As Oyama notes, information is just "a way of talking about certain interactions rather than their cause or a prescription for them" (Oyama 2000, 197).

The contingency and multifactoriality of development challenge the view that teleology and normativity are inherent in biological causation. Instead of there being "right" and "wrong" ways to decode a sequence of 
nucleotides, there are just different causal outcomes that can result from different combinations of interacting factors. For example, at the level of genetic replication, we can think of "replication errors" not as literal mistakes, but as different causal outcomes of the interactions between nucleotides and polymerases due to the influences of external factors and variations in intracellular conditions, much like how the deviations from $F$ $=m \times a$ in the trolley experiment are different outcomes that result from differences in the experimental conditions. At the level of phenotypic development, we can think of different outcomes not as expressions of the genotype gone "right" and gone "wrong", but as different contingent forms that result from different developmental conditions. For example, genetically indistinguishable specimens from the fish species Salmo trutta can develop into the small freshwater brown trout or into the large saltwater sea trout, depending on the ecological conditions in their early developmental stages (Charles et al. 2005). These forms are morphologically and behaviourally different, but are both capable of thriving and reproducing. Neither form represents the "right" way to realise the Salmo trutta genome, but rather both are different causal outcomes that result from different combinations of developmental resources.

At this point, it might be contended that it is possible to discern "right" and "wrong" ways for biological systems to develop by considering whether or not parts of these biological systems are performing their functions. For example, a "replication error" that occurs during genetic replication may be considered to be an instance of the system going "wrong" if it compromises the ability of the resulting cell to function properly. However, this would be to concede that teleology and normativity are instrumental metaphors we project onto biological processes rather than properties of the processes themselves. As Matthew Ratcliffe notes, functions are not found out there in the world, but are contributions to goals "which are themselves instrumentally assigned" (Ratcliffe 2000, 124). That is to say, we instrumentally assign goals to systems and then assign functions relative to those goals. Parts of the systems are deemed to be functional if their effects are conducive to achieving these assigned goals in appropriate ways and are deemed to be dysfunctional if they are failing to produce these effects.

Usually, in biological enquiry, the assigned goal is survival of the biological system. Assigning this goal provides a focus which facilitates questions such as "what is it that x does to contribute to survival?" and "how did it come to do this?" (Ratcliffe 2000, 129). The former question is typically associated with Robert Cummins' (1975) functional analysis of the causal roles of parts of systems, while the latter question is typically 
associated with Ruth Millikan's (1984) aetiological account of function based on the adaptive benefits of the effects of the parts in the evolutionary histories of organisms. While these questions are arrived at through the prior instrumental assignment of a goal, the answers can be expressed in causal and historical terms that do not invoke teleology. For example, we may assign an organism's retina the function of light transduction, because light transduction is the effect of the retina that contributes to the assigned goal of survival. From here, we might go on to explain how light transduction increases the likelihood of survival by influencing the organism's interaction with the environment. We might also go on to explain how the retina came to transduce light by giving a causal account of how past organisms with cells that transduced light had higher chances of producing offspring than past organisms without these cells, which resulted in the evolutionary transmission of the capacity for light transduction to the present organism. The assignment of function provides a focus, but the subsequent explanations are causal and historical explanations that do not themselves invoke a future goal or desired outcome. The normative notions of function and dysfunction, then, are not properties of the causal processes themselves, but are judgements we make relative to the goals we assign.

To further illustrate the instrumentality of function ascription in biology, consider the example of an alteration in an oncogene caused by exposure to an environmental carcinogen. The altered oncogene causally contributes to the accelerated proliferation of malignant tissue containing the altered genotype, which results in tumour progression. Usually, we would consider the alteration in the oncogene to be a dysfunction relative to the assigned goal of survival of the organism. However, it is at least theoretically possible to consider it to be properly functional if a different goal is assigned at a different level of analysis. For example, if we focus on the level of the tumour instead of the level of the organism, then we could claim that the function of the altered oncogene is the proliferation of malignant tissue, insofar as this is the effect of the altered oncogene that contributes to maintenance and progression of the tumour. Furthermore, this could be supported by the aetiological account of function, as the accelerated proliferation of malignant tissue is the effect of the altered oncogene that resulted in the abundance of the altered genotype in the developing tumour. Nonetheless, we tend not to consider the proliferation of malignant tissue to be the function of an altered oncogene, because we tend not to assign a goal at the level of the tumour. Rather, we tend to ascribe the goal of survival at the level of the organism and, accordingly, to consider the proliferation of malignant tissue to be a dysfunction relative to this goal. Hence, as Valerie Hardcastle notes, the assignment of function 
is influenced by a value judgement about which level of analysis is "worthy of teleological language" (Hardcastle 2002, 149).

And so, teleology and normativity are not intrinsic properties of biological processes themselves, but are instrumental metaphors we project onto the biological processes. Biological systems are judged to go "right" or "wrong" relative to goals we assign to them. These normative notions and instrumental goals are derived from our understandings of genuine normativity and teleology in the psychological and social domains. For example, we consider survival of the organism, but not the progression of a tumour, to be a goal, partly because we judge surviving to be valuable and instrumental to our attaining our personal and collective aims and interests. As noted earlier, the informational and semantic notions that are employed in biological theorising are also derived from our understandings of information transfer and semantic content in the social and psychological domains.

The above poses a problem for the account of biopsychosocial causation presented by Bolton and Gillett (2019), because it suggests that normativity and information transfer cannot serve as the common currency in the causal interactions across these three domains. Information transfer and normativity are features of the psychological and social domains respectively, as these involve meanings, intentions, values, and interests. While we may invoke these notions in biological theorising, their uses are metaphorical and do not involve any ontological commitment to the claim that normativity and informational content are properties of the biological systems themselves. Hence, there is no good reason to suppose that the normative and informational notions we invoke in biological explanations refer to the same sorts of normativity and information transfer that feature in social and psychological explanations. There remains a disunity between the interpersonal level and the subpersonal level.

This brings us to the question of whether or not the above undermines the prospect of a philosophically defensible version of the biopsychosocial model. I argue that it does not. Recall that Bolton and Gillett present their account of biopsychosocial causation in order to accommodate the roles of multiple and diverse factors in disease causation while avoiding the physicalistic reductionism of the biomedical model. Accordingly, they suggest that biological, psychological, and social processes are normative processes that regulate one another through information transfer. However, there is no need for Bolton and Gillett to rely on such a metaphysically contested thesis in order to make sense of biopsychosocial causation. The fact that social factors causally influence biological outcomes is uncontroversial in contemporary healthcare and epidemiological research 
has been able to demonstrate these causal relations without having to assume stronger metaphysical claims about biological processes.

Indeed, there is a more established philosophical account of causation that is more metaphysically neutral and can accommodate the roles of diverse factors. This is Woodward's (2004) interventionist theory of causation, which proposes the following:

A necessary and sufficient condition for $X$ to be a (type-level) direct cause of $Y$ with respect to a variable set $\mathbf{V}$ is that there be a possible intervention on $X$ that will change $Y$ or the probability distribution of $Y$ when one holds fixed at some value all other variables $Z_{i}$ in $\mathbf{V}$. (Woodward 2004, 59)

That is to say, causation is analysed as a probabilistic counterfactual dependence relation, wherein $X$ is a cause of $Y$ if and only if an intervention that changes $X$ makes a difference to the probability of $Y$ given appropriate background conditions. Importantly, no ontological restrictions are placed on what sorts of factors can be difference makers. Causal relations between factors can be established by using interventions to demonstrate probabilistic dependencies between the factors, regardless of the organisational levels to which these factors belong. Accordingly, the interventionist theory of causation can accommodate causal relations between factors across biological, psychological, and social domains.

Bolton and Gillett do cite Woodward's interventionist theory of causation in their book. Specifically, they suggest that the interventionist theory of causation is consistent with their claims about agency and causation, insofar as it "emphasises that our interests in causal connections and explanations are linked to our practical concerns of being able reliably to bring about changes" (Bolton and Gillett 2019, 83). The problem, however, is that accepting the interventionist theory of causation makes their metaphysical claims about the normativity and informational content of biological processes somewhat superfluous. As John Campbell (2016) notes, if we understand causal relations in terms of probabilistic dependencies between factors that can be analysed counterfactually, then we do not need to commit to such stronger metaphysical claims in order to make sense of how biological, psychological, and social factors can interact in disease causation. Of course, further scientific research may later yield hypotheses about the mechanisms involved in some, though maybe not all, of these causal relations, but such mechanistic details are not necessary to establish that the factors are causally related. 
The interventionist theory of causation also rejects the physicalistic reductionism of the biomedical model. By understanding causal relations in terms of probabilistic dependencies between factors, psychological and social factors can be acknowledged as genuine causal factors that make differences to biological outcomes, while also accepting that these psychological and social factors may be irreducible to biological processes. For example, recall the various social, political, and economic factors that Bolton and Bhugra (2020) suggest to be contributors to the increasing rates of mental health problems among young people. We can understand these factors as being causal in virtue of how changes in them make differences to the health outcomes when other variables are held fixed. David Stuckler and Sanjay Basu (2013) demonstrate such a causal relation between government austerity and an increase in the population suicide rate by comparing this situation to contrastive scenarios where different policies are associated with different outcomes. Here, establishing such a causal relation requires neither any attempt to reduce government austerity to a different explanatory level, nor any ontological commitment to some deeper property that is conserved or transmitted throughout the causal process.

\section{The Problem of Causal Selection}

The discussion so far suggests that biopsychosocial causation does not have to be so metaphysically taxing. It is widely accepted that social factors can influence biological outcomes and the interventionist theory of causation allows us to make sense of this without having to commit to further ontological claims about the normativity or informational content of biological causation. This raises the question of whether Bolton and Gillett (2019) have misdiagnosed the problem with the traditional version of the biopsychosocial model.

As noted earlier, Ghaemi (2010) criticises the biopsychosocial model for being too vague and too eclectic to have any explanatory value. Such eclecticism, he suggests, was "meant to free practitioners to do what they pleased" (Ghaemi 2010, 213). However, the problem raised by this criticism is not that the biopsychosocial cannot make sense of how the three domains interact causally, but rather that it includes so many causal factors that it does not offer a precise explanation. Alex Broadbent raises a similar worry about the multifactorial model of disease, noting that " $[\mathrm{b}]$ are multifactorialism does nothing to encourage the move from a catalogue of causes to a general explanatory hypothesis" (Broadbent 2009, 307). That is to say, listing more causal factors and causal relations does not necessarily make a model more explanatory. 
The challenge when developing a defensible version of the biopsychosocial model, then, is not so much providing an adequate account of biopsychosocial causation, but providing an adequate account of causal selection. As Broadbent (2009) notes, under the conventional philosophical view of causation, almost every event that is caused is the outcome of multiple causal factors. Nonetheless, we only consider some of these causal factors to be relevant in an explanation. For example, when we want an explanation of house fire, we consider the electrical fault and the building's cladding to be explanatorily relevant, but not the presence of oxygen in the atmosphere, even though the accident was also causally dependent on this. Likewise, given that the biopsychosocial model does not exclude any sorts of causal factors a priori, it is trivially true that every disease is caused by multiple biological, psychological, and social factors. However, this does not tell us which of these factors are relevant in an explanation of the disease.

To some extent, the question of which causal factors are explanatorily relevant is an empirical issue, as we might be able to demonstrate empirically that different cases instantiate different combinations of causal factors. However, it is also to a significant extent a superempirical issue, as we still need to judge which of the many causal factors instantiated by a given case are explanatorily relevant and which comprise the background conditions. For example, we can catalogue all of the causal factors that contribute to a person's type II diabetes mellitus, including insulin resistance, altered $\beta$-cell activity, learned eating behaviour, sedentary labour, economic inequality, and the structure of the food environment, but cataloguing these factors will not inform us which of these factors are deemed explanatory and which are deemed to be in the background, nor will it inform how we should approach the problem. By contrast, the biomedical model fails for dismissing psychological and social factors, but offers a more specific guide to explanation and intervention, insofar as it privileges the biological level as the proper level of analysis.

There are two possible ways in which we might enhance the explanatory power of the biopsychosocial model. The first potential approach is to supplement the biopsychosocial model with a conceptual criterion for selecting explanatory factors from background factors. For example, factors may be deemed more explanatory based on causal proximity, speed of response, or specificity of response (Ross 2018). However, the problem with this approach is that setting a priori constraints on what factors are privileged as explanatorily relevant would revert back to a form of reductionism that the biopsychosocial model is seeking to avoid. Indeed, the physicalistic reductionism of the biomedical model could be interpreted as its assumption of biological proximity as a conceptual 
criterion for which factors are deemed explanatory. Also, a further problem with this approach is that it ignores the different contexts in which different factors might be deemed explanatorily relevant. In different settings, the most explanatorily relevant factors may not be the most proximal, the fastest, or the most specific factors. For example, in a public health context, poor sanitation may be considered a very explanatorily relevant cause of cholera, even though it is not the most proximal cause, the cause with the fastest action, or a cause that is specific to cholera.

This brings us to the second potential approach. This is to acknowledge that which causal factors are deemed explanatory and which are deemed to be in the background are dependent on contexts, values, and interests. As Peter Lipton (2004) notes, explanations are not tout court, but are relative to contrastive foils. For example, when we ask "why did the leaves turn yellow?", the relevant answer will differ depending on whether we are asking "why did the leaves turn yellow in November rather than in January?" or "why did the leaves turn yellow rather than blue?" (Lipton 2004,33 ). This suggests that in order for the biopsychosocial model to be explanatorily useful, we have to be more explicit about our explanatory interests and more specific about the questions we ask. Instead of asking what causes a disease tout court, we can yield more precise causal explanations by considering which contrastive foils are appropriate in the contexts and by asking more specific questions relative to these contrastive foils.

As well as being informed by epistemic and pragmatic considerations, our explanatory interests are often informed by ethical and political considerations, especially in healthcare, where promoting people's welfare and alleviating their suffering are central values. For example, in their recent research on transgender mental health, Sav Zwickl and colleagues apply a psychosocial approach to examine the causal factors associated with suicidality among transgender and nonbinary adults (Zwickl et al. 2021). The context of this research pertains to the higher rates of suicidality and mental health problems among transgender and nonbinary people than among cisgender people, and so the explanatory interests guiding the research are appropriately informed by ethical and political considerations concerning health inequity, social injustice, and systemic discrimination. Guided by these explanatory interests, the researchers were able to discern causal factors for suicidality that disproportionately or specifically affect transgender and nonbinary people, including lack of access to gender affirming healthcare, institutional discrimination, and transphobic violence. These causal factors could have been missed had different explanatory interests guided the research, such as a more general emphasis on the aetiology of mental illness rather than a more specific emphasis on 
the mental health disparities between transgender people and cisgender people.

The above suggests that the biopsychosocial model complements a form of explanatory pluralism in healthcare. Given that it places no a priori constraints on what domains can be causal, it allows for a range of contexts that may require different explanatory approaches. This is noted by Leen De Vreese and colleagues, who suggest that the question "why did person P develop lung cancer?" can allow for many relevance relations, including the following:

(a) Why did person P, who smokes, develop lung cancer, while person P', who also smokes, did not?

(b) Why did person $\mathrm{P}$ with behavior B develop lung cancer, while person P' with behavior B' did not?

(c) Why did person $\mathrm{P}$ living in country $\mathrm{C}$ develop lung cancer, while person P' in country C' did not? (De Vreese et al. 2010, 375-376)

The different relevance relations warrant explanations that appeal to causal factors from different domains. Question (a) is about how a physiological difference between the two people results in smoking having different effects, and so calls for a physiological explanation that draws on biological factors. Question (b) is about the difference between the behaviours of the two people, and so calls for a behavioural explanation that draws on psychological factors. Question (c) is about the effects of the different environments of the two people, and so calls for an epidemiological explanation that draws on social factors.

In turn, the answers to these questions can inform preventative and therapeutic interventions across different healthcare disciplines. For example, the answer to (a) could inform targeted screening and oncological treatment, the answer to (b) could inform behavioural and cognitive interventions such as smoking cessation therapy and motivational counselling, and the answer to (c) could inform public health interventions such as smoking policies and clean air strategies. And so, if we are explicit about our explanatory interests and ask appropriately specific questions, the biopsychosocial model can support clinical interventions that target causal factors across multiple domains.

Of course, explanatory pluralism is not a new idea in the philosophy of medicine. For example, Kenneth Kendler (2005) and Sandra Mitchell 
(2009) have endorsed pluralistic approaches to explaining mental disorders that consider causal factors at genetic, neurobiological, psychological, interpersonal, and cultural levels. However, while the form of explanatory pluralism endorsed by Kendler and Mitchell is an integrative pluralism that seeks to integrate the diverse causal factors at multiple levels into a single comprehensive model, the form of explanatory pluralism I am proposing does not require such integration. Rather, given the biopsychosocial model's wide interdisciplinary scope, it may sometimes be better complemented by a looser form of ineliminative pluralism akin to that suggested by Helen Longino (2013) for studying behaviour. That is to say, we may understand disease causation better by utilising multiple partial accounts than by attempting to assemble a more general model that incorporates all the causal factors. Different partial accounts may be relevant to different explanatory interests and may draw on different sets of causal factors. For example, in response to the aforementioned question "why did person P develop lung cancer?", whether we consider a predominantly physiological account, a predominantly behavioural account, or a predominantly epidemiological account to be appropriate will depend on the relevance relations in which we are interested (De Vreese $e t$ al. 2010). It may not be possible to integrate these accounts into a single comprehensive model that represents all of the causal relations between the different domains, but this does not compromise the clinical value of the biopsychosocial model.

\section{Conclusion}

Bolton and Gillett (2019) are correct that there is good reason to endorse the biopsychosocial model in contemporary healthcare. Given the substantial evidence of social causation and the problem with physicalistic reductionism, the biomedical model is untenable as a regulative ideal for medicine. And so, a broad biopsychosocial approach is required to accommodate the diverse range of factors involved in disease causation and to inform interventions on these factors across multiple domains.

The criticism that the biopsychosocial model is too vague to be explanatorily valuable is taken by Bolton and Gillett to suggest that the traditional version of the model lacks an appropriate account of biopsychosocial causation. Accordingly, they present a metaphysical account of biopsychosocial causation that suggests that normative processes in the biological, psychological, and social domains regulate one another through information transfer. Herein, I have raised some problems with their account and have argued that the issue of biopsychosocial causation does not have to be so metaphysically taxing, as the causal 
relations between factors in the different domains can be accommodated by the more metaphysically neutral interventionist theory of causation. Furthermore, I have argued that the purported vagueness of the biopsychosocial model is not due to the issue of biopsychosocial causation, but is due to the issue of causal selection. Nonetheless, this can easily be overcome being more explicit about our explanatory interests in different contexts and more specific about the questions we ask. When this pluralistic approach to explanation is applied, the eclecticism of the biomedical model is shown not to be its weakness, but its principal strength.

\section{Acknowledgments}

I would like to thank Awais Aftab for his very generous advice on this paper and the two anonymous reviewers for taking the care to offer constructive comments. I am grateful to the Leverhulme Trust for supporting this research through an Early Career Fellowship (grant reference ECF-2017-298).

\section{REFERENCES}

Bolton, Derek, and Dinesh Bhugra. 2020. 'Changes in Society and Young People's Mental Health'. International Review of Psychiatry, DOI: $10.1080 / 09540261.2020 .1753968$.

Bolton, Derek, and Grant Gillett. 2019. The Biopsychosocial Model of Health and Disease. Cham: Palgrave Macmillan.

Broadbent, Alex. 2009. 'Causation and Models of Disease in Epidemiology'. Studies in History and Philosophy of Biological and Biomedical Sciences, 40: 302-311.

Campbell, John. 2016. 'Validity and the Causal Structure of a Disorder'. In Philosophical Issues in Psychiatry IV: Psychiatric Nosology, edited by K. S. Kendler and J. Parnas, 257-273. Oxford: Oxford University Press.

Cartwright, Nancy. 1983. How the Laws of Physics Lie. Oxford: Clarendon Press.

Charles, Katia, Guyomard, René, Hoyheim, Björn, Ombredane, Dominique, and Jean Luc Baglinière. 2005. 'Lack of Genetic Differentiation Between Anadromous and Resident Sympatric Brown Trout (Salmo trutta) in a Normandy Population'. Aquatic Living Resources, 18: 65-69.

Cummins, Robert. 1975. 'Functional Analysis'. Journal of Philosophy, 72: 741-765. 
Dawkins, Richard. 1995. River Out of Eden: A Darwinian View of Life. New York: Basic Books.

De Vreese, Leen, Weber, Erik, and Jeroen Van Bouwel. 2010. 'Explanatory Pluralism in the Medical Sciences: Theory and Practice'. Theoretical Medicine and Bioethics, 31: 371-390.

Ghaemi, Nassir. 2010. The Rise and Fall of the Biopsychosocial Model: Reconciling Art and Science in Psychiatry. Baltimore, MD: Johns Hopkins University Press.

Griffiths, Paul E., and R. D. Gray. 1994. Developmental Systems and Evolutionary Explanation. Journal of Philosophy, 91: 277-304.

Hardcastle, Valerie. G. 2002. 'On the Normativity of Functions'. In Functions: New Essays in the Philosophy of Psychology and Biology, edited by A. Ariew, R. Cummins, and M. Perlman, 144156. New York: Oxford University Press.

Kendler, Kenneth S. 2005. 'Toward a Philosophical Structure for Psychiatry'. American Journal of Psychiatry, 162: 433-440.

Lakatos, Imre. 1974. 'Science and Pseudoscience'. Conceptus, 8: 5-9.

Lipton, Peter. 2004. Inference to the Best Explanation, $2^{\text {nd }}$ edition. London: Routledge.

Longino, Helen. 2013. Studying Human Behavior: How Scientists Investigate Aggression and Sexuality. Chicago: University of Chicago Press.

Marmot, Michael. 2005. 'Remediable or Preventable Social Factors in the Aetiology and Prognosis of Medical Disorders'. In Biopsychosocial Medicine: An Integrated Approach to Understanding Illness, edited by P. D. White, 39-58. New York: Oxford University Press.

Millikan, Ruth G. 1984. Language, Thought and Other Biological Categories: New Foundations for Realism. Cambridge, MA: MIT Press.

Mitchell, Sandra D. 2009. Unsimple Truths: Science, Complexity, and Policy. Chicago: University of Chicago Press.

Newen, Albert, De Bruin, Leon, and Shaun Gallagher. 2018. 4E Cognition: Historical Roots, Key Concepts, and Central Issues. In The Oxford Handbook of $4 E$ Cognition, eds. A. Newen, L. De Bruin, and S. Gallagher, 3-18. Oxford: Oxford University Press.

Oyama, Susan. 2000. The Ontogeny of Information: Developmental Systems and Evolution. Durham, NC: Duke University Press.

Plomin, Robert. 2018. Blueprint: How DNA Makes Us Who We Are. Cambridge, MA: MIT Press.

Ratcliffe, Mathew. 2000. 'The Function of Function'. Studies in History and Philosophy of Biological and Biomedical Sciences, 31: 113133. 
Ross, Lauren N. 2018. 'Causal Selection and the Pathway Concept'. Philosophy of Science, 85: 551-572.

Schrödinger, E. 1944. What is Life? Cambridge: Cambridge University Press.

Stuckler, David., and Sanjay Basu. 2013. The Body Economic: Why Austerity Kills. London: Allen Lane.

Woodward, John. 2004. Making Things Happen: A Theory of Causal Explanation. Oxford: Oxford University Press.

Zwickl, Sav, Wong, Aalex Fang, Dowers, Eden, Leemaqz, Shalem YinerLee, Bretherton, Ingrid, Cook, Teddy, Zajac, Jeffrey D., Yip, Paul S. F., and Ada S. Cheung. 2021. 'Factors Associated with Suicide Attempts Among Australian Transgender Adults'. BMC Psychiatry, 21: 81 . 
